Richard A. Miller

Scott B. Berger

David T. Burke

Andrzej Galecki

Gonzalo G. Garcia

James M. Harper

Amir A. Sadighi Akha

\section{T cells in aging mice: genetic, developmental, and biochemical analyses}

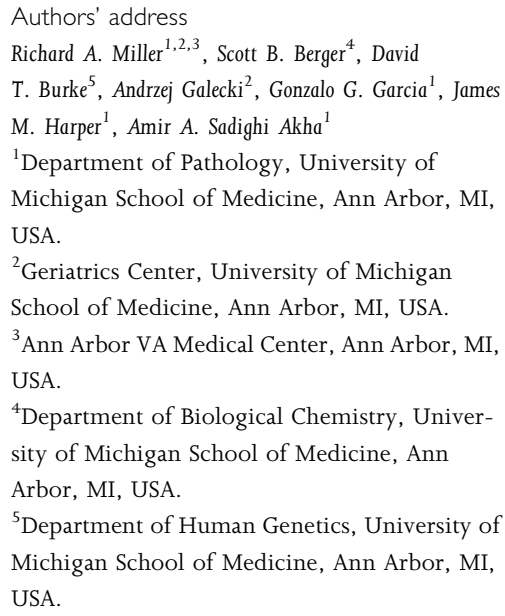

Immunological Reviews 2005

Vol. 205: 94-103

Printed in Singapore. All rights reserved

Copyright (C) Blackwell Munksgaard 2005

Immunological Reviews

0105-2896
Summary: A combination of approaches - gene mapping, biomarker analysis, and studies of signal transduction - has helped to clarify the mechanisms of age-related change in mouse immune status and the implications of immune aging for late-life disease. Mapping studies have documented multiple quantitative trait loci (QTL) that influence the levels of age-sensitive T-cell subsets. Some of these QTL have effects that are demonstrable in young-adult mice ( 8 months of age) and others demonstrable only in middle-aged mice (18 months). Biomarker studies show that T-cell subset levels measured at 8 or 18 months are significant predictors of lifespan for mice dying of lymphoma, fibrosarcoma, mammary adenocarcinoma, or all causes combined. Mice whose immune systems resemble that of young animals, i.e. with low levels of $\mathrm{CD}^{+}$ and $\mathrm{CD}^{+}$memory $\mathrm{T}$ cells and relatively high levels of $\mathrm{CD} 4^{+} \mathrm{T}$ cells, tend to outlive their siblings with the opposite subset pattern. Biochemical analyses show that $\mathrm{T}$ cells from aged mice show defects in the activation process within a few minutes of encountering a stimulus and that the defects precede the recognition by the T-cell receptor of agonist peptides on the antigen-presenting cell. Defective assembly of cytoskeletal fibers and hyperglycosylation of T-cell surface glycoproteins contribute to the immunodeficiency state, and indeed treatment with a sialylglycoprotein endopeptidase can restore full function to $\mathrm{CD} 4^{+} \mathrm{T}$ cells from aged donors in vitro.

\section{Introduction}

The immune system is the gerontologist's playground. Immune systems fail with age in clinically significant ways, can be dissected in vitro and re-assembled in vivo, and are the obsession of legions of professional immunologists who, because they do not care that much about aging, are often willing to share cell lines, mouse stocks, antibodies, and ideas. This article presents illustrations of ways in which the momentum and accomplishments of the immunological community 
can be brought to bear on three problems in aging research: genetic control of late-life changes, pathobiology of aging and cancer, and the cell biology of immune failure.

\section{Genetic dissection of T-cell subset changes in aging mice}

Our genetic analyses employ mice of a stock called UM-HET3, created by a four-way cross in which CB6F1 females are mated to $\mathrm{C} 3 \mathrm{D} 2 \mathrm{~F} 1$ males. The individual animals are the genetic equivalent of full siblings; no two pups are the same. Each individual shares a random $50 \%$ of its genetic endowment with every other animal in the population, receiving approximately $25 \%$ of its alleles from each of the four grandparental strains, BALB/cJ, C57BL/6 J, C3H/HeJ, and DBA/2 J. DNA from each mouse is genotyped at 100-300 polymorphic alleles to acquire a moderate resolution picture of which chromosomal segments it has inherited from each of the four grandparents. Each mouse is then evaluated for phenotypic traits of interest, for example, levels of age-sensitive T-cell subsets, and a permutation-adjusted regression analysis then looks for loci at which one of the alleles has a greater than random level of association with a trait of interest.

We have used these methods $(1,2)$ to document genetic controls of seven T-cell subsets: $\mathrm{CD}^{+}$and $\mathrm{CD} 8^{+}$cells, $\mathrm{CD} 4^{+}$ and $\mathrm{CD}^{+}$memory cells (using $\mathrm{CD} 44$ level as an index of memory cell differentiation; abbreviated CD4M and CD8M), $\mathrm{CD}^{+}$naïve cells (using CD45RB as index), and subpopulations of $\mathrm{CD}^{+}$and $\mathrm{CD} 8^{+}$cells that express P-glycoprotein (referred to as CD4P and CD8P). These latter two subsets are of special interest, because their proportions increase dramatically with age (3), even in naïve $\mathrm{T}$ cells in $\mathrm{T}$-cell receptor (TCR) transgenic ( $\mathrm{Tg}$ ) mice in which conversion of naïve to memory cells is greatly slowed (4), and because CD4P cells are anergic in tests of proliferation, cytokine production, and calcium signal generation (5-7). Catalogs of alleles that modulate each of these T-cell subsets $(1,2)$ are now available, and they will be of use in studies of genetic controls of late-life immune function and susceptibility to neoplastic and autoimmune illnesses and immunodeficiency states. Here, we use them to illustrate two broader themes: (i) stage-specific effects on age-sensitive traits and (ii) use of composite indices of agedependent T-cell patterns.

The top panels of Fig. 1 show examples of loci that influence age-sensitive T-cell subsets, but they do so through actions that affect mice in the first third of the lifespan and remain consistent at least through 18 months of age. The upper left panel, for example, shows that mice that inherit the B6 allele linked to the chromosome 12 marker D12Mit105 tend to have higher levels of CD8M cells. This difference is apparent in mice evaluated at 8 months of age, and it is still present, and to the same degree, when the mice are tested at 18 months of age. The upper right panel shows another genetic polymorphism between BALB/c and B6 mice, this one linked to the D12Mit34 marker, which modulates CD4M levels at both ages tested. In this case, it is the BALB/c allele that is associated with the higher memory cell levels. In both these cases and in others tabulated in the references cited, the levels of the T-cell subsets differ in young adult mice and then shift in an alleleindependent way as the mice get older. In contrast, the bottom two panels of Fig. 1 show examples of loci where polymorphisms have no influence on subset levels in young adults (i.e. 8-month-old mice), but instead modify the rate at which the subsets change in the ensuing 10-month period. The left panel shows the effect of a chromosome 9 quantitative trait locus (QTL) on CD4P cell levels, in which the polymorphism is inherited from the $(\mathrm{C} 3 \mathrm{H} \times \mathrm{DBA} / 2) \mathrm{F} 1$ parent, and the right panel shows a maternal locus that influences the rate of change of $\mathrm{CD}^{+}$cells in aging mice. Similar analyses have been used to map polymorphisms that regulate age-sensitive B-cell subsets in the same population of UM-HET3 mice (Labrie et al., manuscript submitted), T-cell proliferation responses (Miller et al., unpublished observations), and a wide range of other phenotypes including hormone levels (8), cataracts (9), bone fragility (10), and lifespan $(11,12)$.

\section{T-cell subset levels as predictors of lifespan and cancer risk}

The experimental protocol used to monitor T-cell subset levels in aging UM-HET3 mice does not require that the mice be killed, and thus it permits a test of the idea that those mice whose immune systems show the most advanced signs of aging are indeed likely to die at earlier ages than those mice with younger looking T-cell subset patterns. There are two underlying models, not mutually exclusive. The 'biomarker' model imagines that individual mice age at different rates, so that mice with faster rates of aging will show more rapid changes in T-cell subset patterns and also more rapid acquisition of lethal diseases, as well as rapid accumulation of other signs and symptoms of aging. The 'immune protection' model imagines that the immune systems of young mice confer protection against a wide range of late-life diseases and that those mice that retain youthful immune systems into old age are thereby protected against ill health.

Initial evidence from a group of 173 mice showed that the proportion of CD4M cells in 18-month-old mice was a significant $(\mathrm{P}<0.003)$ predictor of longevity in the UM-HET3 population (13); several other subsets showed suggestive 

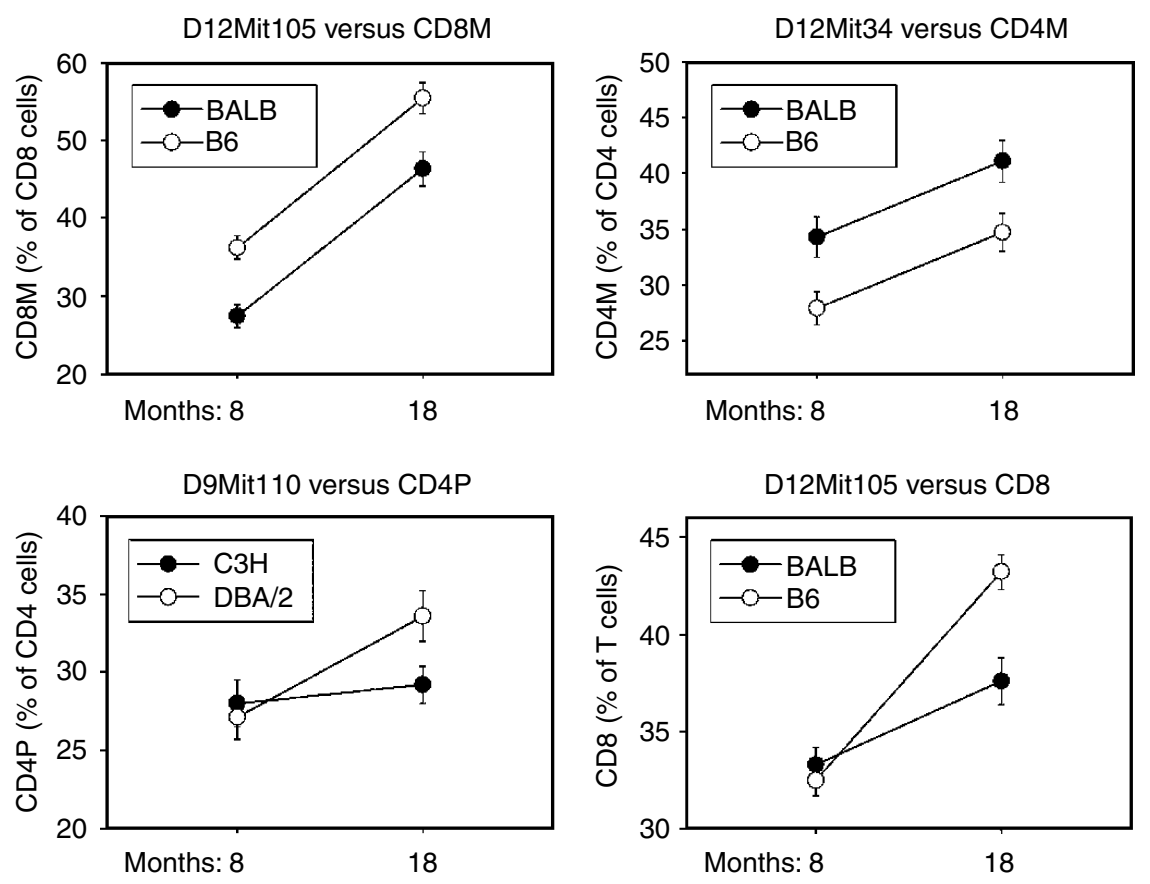

Fig. 1. Illustrations of quantitative trait loci (QTL) that modulate T-cell subset levels in UM-HET 3 mouse populations. Four QTL are shown, two (top rows) whose influence on subset levels is apparent in 8-month-old mice and is maintained at least through 18 months, and two others (bottom row) whose effects are not apparent until 18 months of age. Each symbol shows mean level of the indicated subset (with standard error) in the population of mice bearing the indicated allele (example: $\mathrm{BALB} / \mathrm{c}$ or B6 alleles for the D12Mit 105 locus shown in the upper right panel). Each of these effects reaches statistical significance using experiment-wise criteria as explained previously $(1,2)$. associations that did not reach statistical significance $(0.05<\mathrm{P}<0.1)$ in this preliminary analysis. Consistent with the working hypothesis, mice with the highest levels of CD4M cells, i.e. mice whose immune system resembled that of older animals, tended to die at relatively early ages. A second report extended this analysis to a total of 559 mice (14). In this larger group, five age-sensitive $\mathrm{T}$-cell subsets among the seven studied were each found to be a significant predictor of lifespan when measured in blood at 18 months of age. In each case, the sign of the regression coefficient was consistent with the hypothesis that mice whose immune system resembled that of younger animals would tend to have longer lifespans. A cluster analysis method showed that when 18-month-old mice were divided into two groups based solely on similarity of T-cell subset patterns, one of the clusters resembled young mice (with relatively low levels of CD4M, CD8M, and CD4P cells and relatively high levels of CD4 and CD4 naïve cells), and that mice in this cluster were long-lived compared to the remainder of the population. The ability of the cluster approach to predict lifespan on the basis of T-cell subset patterns was equally apparent in virgin male mice, virgin females, and females that had given birth to multiple litters as young adults, and can thus be considered to be replicated in these three nonoverlapping groups.

A statistical approach based on principal components analysis was then applied to this data set in an attempt to develop an overall index of age-sensitive T-cell subset pattern changes that might be more informative than looking at each $\mathrm{T}$-cell subset separately (15). The algorithm forms a composite index as a weighted average of the values of each of the seven measured T-cell subsets, with weights chosen so as to maximize the amount of variation explained by the calculated index among the mice in the population. The calculated 'immune subset factor' was correlated strongly with the age-sensitive subsets, and indeed mice with high values of the calculated factor were those with relatively high $\mathrm{CD} 8, \mathrm{CD} 4 \mathrm{M}, \mathrm{CD} 8 \mathrm{M}$, and CD4P levels, and relatively low CD4 and CD4 naïve populations; in other words, mice with high-factor scores looked older in immunological terms. We then tested the prediction that mice with high levels of this factor would die young. Fig. 2 shows the relationship of principal component scores (termed 'F1_18' to indicate that it is calculated from data obtained at 18 months of age) to age at death in groups of mice that died of each of the three most common lethal illnesses, lymphoma (left), mammary adenocarcinoma (middle), and fibrosarcoma (right). Mice with young looking immune systems (low levels of the factor score) were likely to survive longer in each of the three diagnostic groups, as well as in the group of mice that died of some other cause (data not shown). The association between factor score and mortality risk was statistically significant for all four population subgroups, and reached $\mathrm{P}<0.0001$ for the mouse population as a whole. Thus these data suggest that mice whose changes in age-related T-cell subset pattern are either delayed or decelerated tend to be healthier in old age and survive longer. The association does not apply merely to a single cause of death, as would be 

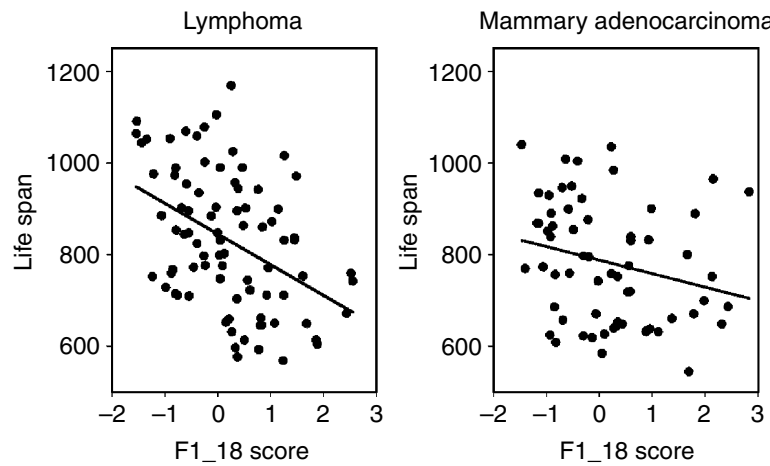

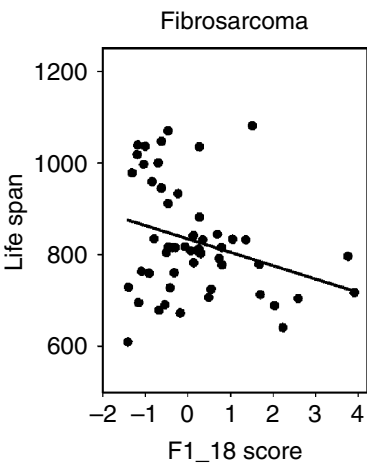

Fig. 2. Plots of mouse lifespan as a function of T-cell subset index (F1_18 score) measured in UM-HET3 mice at 18 months of age. Three separate groups of mice are shown, representing (left to right) those destined to die of lymphoma, mammary adenocarcinoma, or fibrosarcoma. High scores of F1_18 represent mice with higher levels of

expected if, for example, preneoplastic changes in T-cell developmental pathways led both to lymphoma and to subset shifts. We favor the idea that mice with relatively rapid immune aging will also show rapid aging in other non-hematopoietic systems, and there is some support for this notion from evidence that aged mice with advanced subset shifts also have relatively weak muscles (16). We cannot yet rule out, however, the immune protection hypothesis that rapid immune aging predisposes to multiple forms of late-life neoplastic disease.

A similar composite index of age-sensitive T-cell subsets was also found, in the same study (15), to be able to predict remaining longevity when measured in mice as young as 8 months of age. These data are consistent with the hypothesis that age-dependent changes in T-cell subsets may be initiated, at least in some mice, at ages less than 8 months of age.

A more recent study (17) evaluated T-cell subsets as predictors of longevity in an independent replicate population of 539 UM-HET3 mice. Three of the tested subsets (CD4, CD8, and CD8M) were found to be significant predictors of lifespan when tested at 18 months of age, and CD8M cells were predictive as early as 8 months of age. A suggestive relationship was also noted for the CD4M subset $(\mathrm{P}<0.07)$. Composite indices calculated by the principal component method were again found to be significant predictors of lifespan, whether evaluated at 8 or 18 months of age. Thus the main findings of the earlier studies (except for results on the CD4P subset, which were not replicated in the most recent population) seem to be fairly robust, at least for the genetically heterogeneous UM-HET3 population.

We have unpublished evidence that CD4M cells are significant predictors of life expectancy in female mice of two wild-derived mouse stocks (18), one derived from mice trapped in Idaho (Id) and the other derived from mice trapped on the Pacific island of
CD4 and CD8 memory cells, lower levels of CD4 cells, and higher levels of CD4 cells with P-glycoprotein expression. Each symbol represents one mouse, and the lines are calculated by linear regression. F1_18 score is significantly associated with differences in longevity for each of the three mouse subpopulations. Additional details shown in Miller \& Chrisp (15).

Majuro (Ma). Fig. 3 shows the relationship between CD4M levels, measured at 18 months of age, and age at death in these two stocks. A Cox regression analysis showed that the risk ratios were respectively 1.58 and 1.61 for all-cause mortality, both significant at $\mathrm{P}<0.03$. Similar results were seen in segregating populations of $\mathrm{F} 2$-hybrid mice created by crosses of Id or Ma to mice of the $\mathrm{C} 57 \mathrm{BL} / 6 \mathrm{~J}$ inbred stock. The risk ratios were $1.42(\mathrm{P}=0.07)$ for the $(\mathrm{Id} \times \mathrm{B} 6) \mathrm{F} 2$ population and 1.79 $(\mathrm{P}=0.02)$ for the $(\mathrm{Ma} \times \mathrm{B} 6) \mathrm{F} 2$ population. In each case, high levels of CD4M cells were associated with an increased mortality risk as in the UM-HET3 studies. We conclude from this finding that the association between high levels of $\mathrm{CD} 4 \mathrm{M}$ cells and increased mortality risk is replicable in multiple mouse populations with different genetic backgrounds, including hybrids between wild-derived and laboratory-derived stocks.

\section{Mapping the genes for age-sensitive immune subset patterns}

Composite indices of T-cell subset patterns, by combining information from multiple, correlated T-cell subset measurements in each animal, could provide a more informative index of agerelated change than would be provided by any one subset tested by itself. Indeed, the data in Fig. 2 show that principal component factors developed in this way are able to predict lifespan, even in the relatively small groups of mice that share a specific cause of death. For this reason, we tested the idea that the composite scores themselves might be under the control of one or more polymorphic genetic loci, using the same methods that we had previously used to map QTL that moderated levels of specific T-cell subsets in young and old mice. In a population of $>500$ UM-HET3 mice, we found evidence for alleles at two loci, D4Mit55 and D13Mit21, each of which had a significant 


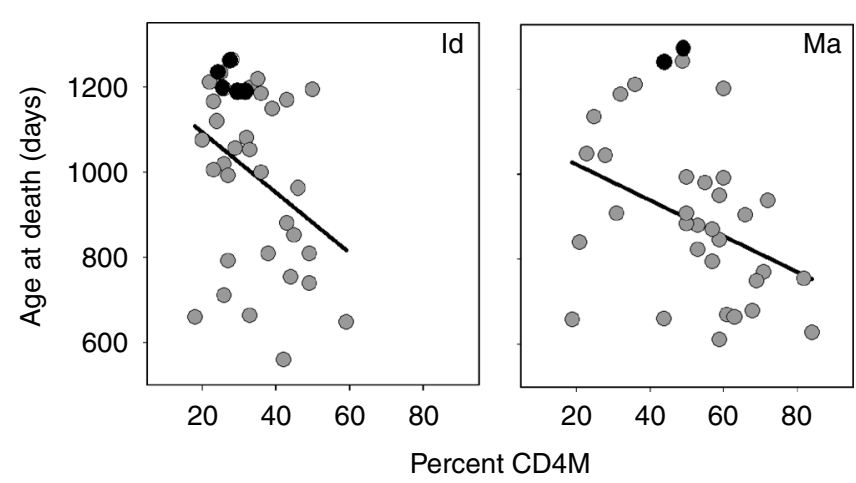

Fig. 3. Prediction of lifespan by CD4M (memory subset) levels measured at 18 months of age in two stocks of wild-derived mice, Idaho (Id, left panel) and Majuro (Ma), shown in previous work (18) to be longer lived than typical laboratory-adapted stocks of inbred and hybrid mice. Each symbol represents one mouse; filled symbols show mice that were still alive as of November 1, 2004. Each association is significant at $\mathrm{P}<0.05$ by log-rank test. Lines are calculated by linear regression.

association with immune subset pattern as expressed in the principal component factor score (2). Interestingly, neither of these loci had reached experiment-wise significance in the QTL screen that evaluated single subsets, consistent with the idea that the factor score, representing a combination of age-sensitive Tcell subsets, might itself be regulated by polymorphisms whose effects on individual subsets were substantially diluted by subset-specific genetic or nongenetic controls. An analysis of a separate population of UM-HET3 mice has provided independent support for the conclusion that genes on chromosomes 4 and 13 exert effects on age-sensitive T-cell subset patterns (17).

\section{T-cell activation defects}

The data presented above show that age-related shifts in T-cell subsets are intimately associated with lifespan and disease risk and that they are under the control of genes that are polymorphic within the commonly used stocks of laboratory mouse, but they provide no insight into the alterations in cell biology that might contribute to diminished immune responses in old age. We have addressed the latter problem by studies of T-cell signal transduction. An earlier review (19) summarized studies through 1997 showing age-related declines in early aspects of T-cell activation including development of calcium signals, phosphorylation of elements of the TCR-CD3 signal transduction complex, and activation of downstream kinases including extracellular-regulated kinase (ERK) and mitogen-activated protein kinase (MAPK)/ERK (MEK). Further work on downstream signals subsequently revealed parallel age-related declines in activation of Raf- 1 kinase (20) and in c-Jun N-terminal kinase function $(21,22)$.

\section{Defects precede TCR-antigen recognition}

A turning point in these studies was the observation that $\mathrm{T}$ cells from young and aged mice had equal amounts of $\zeta$-associated protein of $70 \mathrm{kDa}$ (Zap-70) enzyme activity, tested in lysates, in the first few minutes after exposure to an activating signal (23); tyrosine-specific phosphorylation of the Zap-70 molecule was equivalent in activated $\mathrm{CD} 4{ }^{+} \mathrm{T}$ cells of both young and old mice. This lack of age effect was surprising in view of the clear evidence for age-dependent differences in most downstream-signal events, including phosphorylation of the Zap-70 substrate linker of activated T cells (LAT) in intact $\mathrm{T}$ cells (24). The paradox was resolved by microscopic analysis, using confocal immune fluorescence methods, of conjugates between $\mathrm{T}$ cells and hybridoma cells presenting anti-CD3 antibody as a polyclonal activator. $\mathrm{CD} 4^{+} \mathrm{T}$ cells from young mice were able to form immunological synapses containing LAT, Vav, protein kinase $\mathrm{C} \theta$ ( $\mathrm{PKC} \theta$ ), and other components of the T-cell signal complex in about half of the conjugates formed with anti-CD3 hybridoma cells as polyclonal antigen-presenting cells (APCs) $(24,25)$, but only $25 \%$ of $\mathrm{CD}^{+}{ }^{+} \mathrm{T}$ cells from aged donors were able to generate immunological synapses. A similar discrepancy was noted in a system in which the $\mathrm{T}$ cells expressed a TCR specific for a peptide antigen derived from pigeon cytochrome $\mathrm{C}$, using peptide-bearing lymphoma cells as APCs (26). Synapse formation was an 'all-or-none' reaction, in the sense that those $\mathrm{T}$ cells from mice of any age that moved LAT into the synaptic region almost always also moved PKC $\theta$ as well and vice versa, and then proceeded to translocate the DNA-binding factor, nuclear factor of activated $\mathrm{T}$ cells into their nucleus as well (24). The ability to form synapses was strongly associated with subsequent steps in T-cell activation, in that those $\mathrm{CD}^{+}{ }^{+} \mathrm{T}$ cells which failed to form synapses were predominantly members of a T-cell subset detectable by expression of the multiple-drug resistance pump P-glycoprotein, of which previous work had shown was functionally anergic (5-7). Thus, these results supported a model in which poor signal transduction in $\mathrm{CD} 4^{+} \mathrm{T}$ cells from aged mice was the result of an accumulation of cells that were unable to form functional synapses necessary for kinase-mediated activation of downstream stages of the activation process.

\section{Defects in cytoskeletal activation}

Where, then, lay the key defect(s) in the synapse formation process? New information came from the observation that the defect in $\mathrm{T}$ cells from aged donors preceded recognition of antigenic peptides by the TCR. The protocol made use of a system in which naïve $\mathrm{CD} 4^{+} \mathrm{T}$ cells from TCR-Tg mice were 
allowed to form conjugates with lymphoma APCs bearing either agonist peptide (derived from pigeon cytochrome $\mathrm{C}$ sequence) or an antagonist peptide that differed from the agonist at a single amino acid. Others had shown previously that antagonist peptides induced a presynaptic response characterized by movement of the cytoskeletal protein talin, but that formation of a fully functional synapse with LAT, Vav, actin, and other components in addition to talin depended on recognition of the agonist peptide sequence (27). Evaluation of talin rearrangements in the TCR-Tg system then showed that while about $60 \%$ of CD4 cells from young mice were able to relocate talin to the APC contact zone in response to agonist or antagonist peptide, the proportion of responsive cells from aged mice was much lower, i.e. about $15 \%$ in response to antagonist peptide and at most $31 \%$ in responses to agonist (28). Fig. 4 shows summary statistics from a series of three experiments using six young and three aged donors (28). The top panel shows the proportion of unresponsive cells in conjugates containing agonist peptide; as expected, $70 \%$ to $80 \%$ of the $\mathrm{CD}^{+} \mathrm{T}$ cells from aged donors are unresponsive whether the reactions are monitored by talin or by actin movement, and in contrast most young $\mathrm{CD}^{+} \mathrm{T}$ cells are responsive to agonist peptide. When antagonist peptide is used (bottom panel), as expected, neither old nor young cells respond by actin relocation ( $>80 \%$ unresponsive cells). The response of talin in antagonist-challenged cells (right bars, lower panel) is much stronger in young cells than in old cells. The failure of $\mathrm{CD} 4^{+} \mathrm{T}$ cells from aged donors to relocate talin to the synapse in response to antagonist peptide shows that at least one age-sensitive step in the activation process involves a step prior to the discrimination, by the TCR, between agonist and antagonist peptides.

This age-sensitive defect in talin relocation focused attention on other aspects of cytoskeletal remodeling in the T-cell activation cascade. An assay in which $\mathrm{CD} 4{ }^{+} \mathrm{T}$ cells from young or old mice were allowed to attach to glass slides showed that slides coated with anti-CD3 (but not with anti-DNP or antiCD28 antibodies) stimulated many T cells to form pseudopodia and spread out onto the glass surface. Such interactions involve reorganization of the cytoskeleton and polymerization of actin into a ring at the edge of the spreading cell, but do not involve construction of a complete immunological synapse. The data in Fig. 5, excerpted from a more comprehensive set of experiments using a range of doses and time points (28), illustrate that $\mathrm{CD}^{+} \mathrm{T}$ cells from aged mice are less likely than those from young mice to spread out after attachment to anti-CD3-coated slides, whether the cells are derived from CB6F1 mice or (right panel, Fig.5) from TCR-Tg mice in which most of the $\mathrm{CD}^{+}{ }^{+} \mathrm{T}$ cells remain in the naïve subset throughout life.

A third line of analysis involved detection of translocation of $\mathrm{CD} 3 \zeta$ chains from the soluble to the cytoskeletal compartment after stimulation of $\mathrm{T}$ cells by cross-linking CD3 to CD4 and CD28. Stimulation of CD4 cells from young mice induced a rapid ( $5 \mathrm{~min}$ ) increase in the amount of $\mathrm{CD} 3 \zeta$ associated with the cytoskeletal fraction, with a corresponding decline in $\mathrm{CD} 3 \zeta$ in detergent-soluble form. $\mathrm{CD}^{+}{ }^{+} \mathrm{T}$ cells from aged donors, however, showed neither effect. Fig. 6 illustrates this difference for the p16 form of $\mathrm{CD} 3 \zeta$, and the original study (28) shows similarly large and significant changes for the p21 and p23 forms of $\mathrm{CD} 3 \zeta$ as well.

All three lines of experiments suggest that $\mathrm{CD} 4^{+} \mathrm{T}$ cells from aged mice show defects in cytoskeletal reorganization after
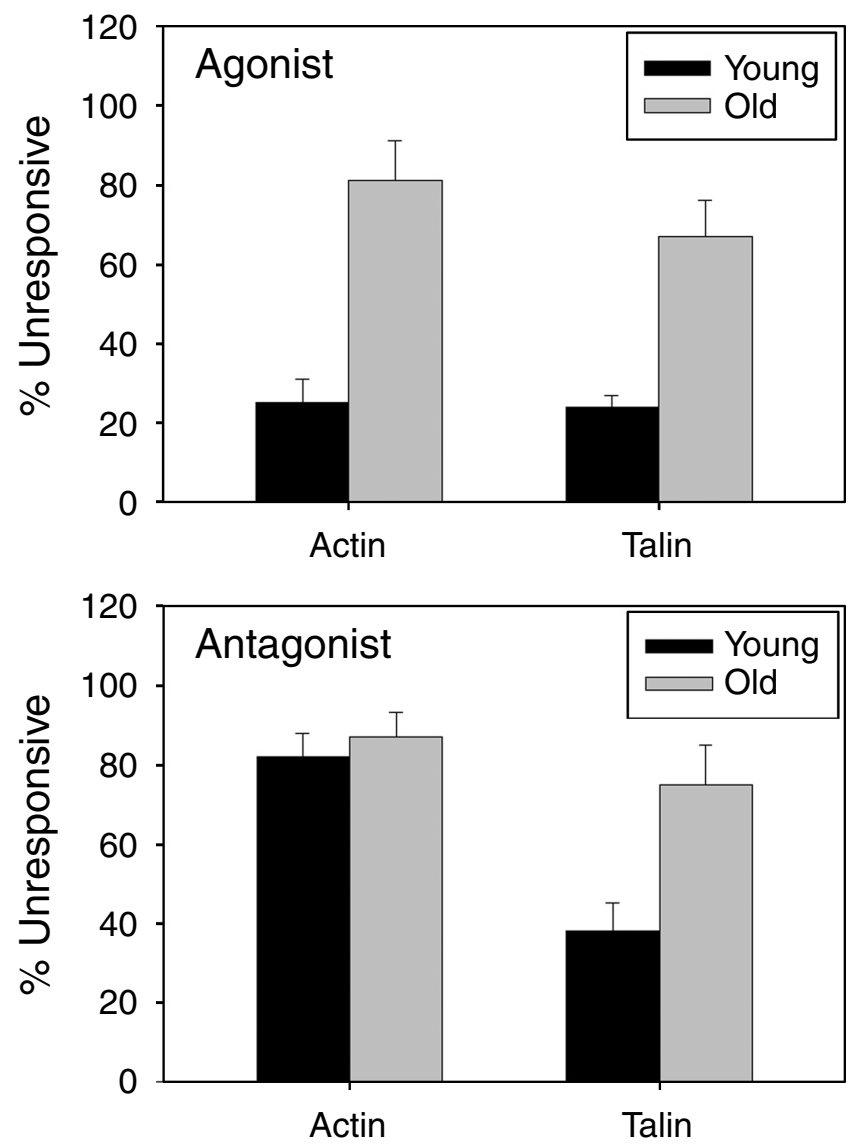

Fig. 4. $\mathrm{CD}^{+} \mathrm{T}$ cells from old mice fail to respond to antigenpresenting cells (APCs) bearing agonist or antagonist peptides. APCs bearing either agonist (PCCF, pigeon cytochrome $\mathrm{C}$ fragment) or antagonist peptides were allowed to form conjugates with $\mathrm{CD} 4{ }^{+} \mathrm{T}$ cells from young or old AND strain T-cell receptor transgenic mice. Confocal immunofluorescent methods were then used to count the proportion of conjugates that moved either actin or talin to the site of T-cell/APC contact. Each bar shows mean and standard error for the proportion of nonresponding cells from six young and three old mice tested in three separate experiments. Data derived from Garcia \& Miller (28). 

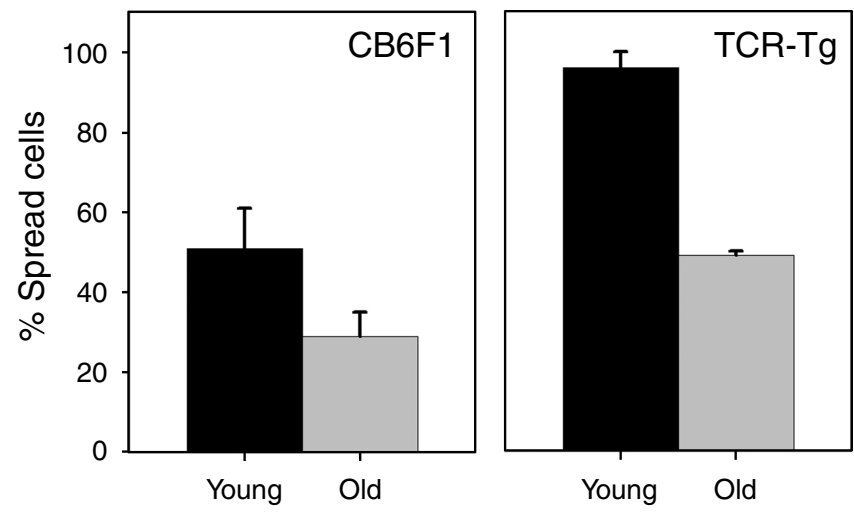

Fig. 5. Percentage of splenic $\mathrm{CD}^{+}{ }^{+} \mathrm{T}$ cells that spread after attachment to glass slides coated with anti-CD3 antibody. Left panel: cells from CB6F1 mice; right panel: cells from AND stock mice transgenic for the pigeon cytochrome $\mathrm{C}$-specific T-cell receptor. Bars show mean and standard errors of the mean for at least three pairs of young and old mice tested on separate days. A cell was scored as positive for spreading if its area, at the point of attachment to the glass, was greater than an area achieved by only $5 \%$ of $\mathrm{T}$ cells from old mice on slides coated with a non-stimulatory control antibody (28).

encounter with TCR-specific stimuli, defects that precede antigen recognition (Fig. 4), do not require interaction with costimulatory molecules present on APCs (Fig. 5) and modulate interaction of CD3 components with the cytoskeleton (Fig. 6). These results are consistent with models in which alterations in TCR-cytoskeletal connections contribute to defects in immu-

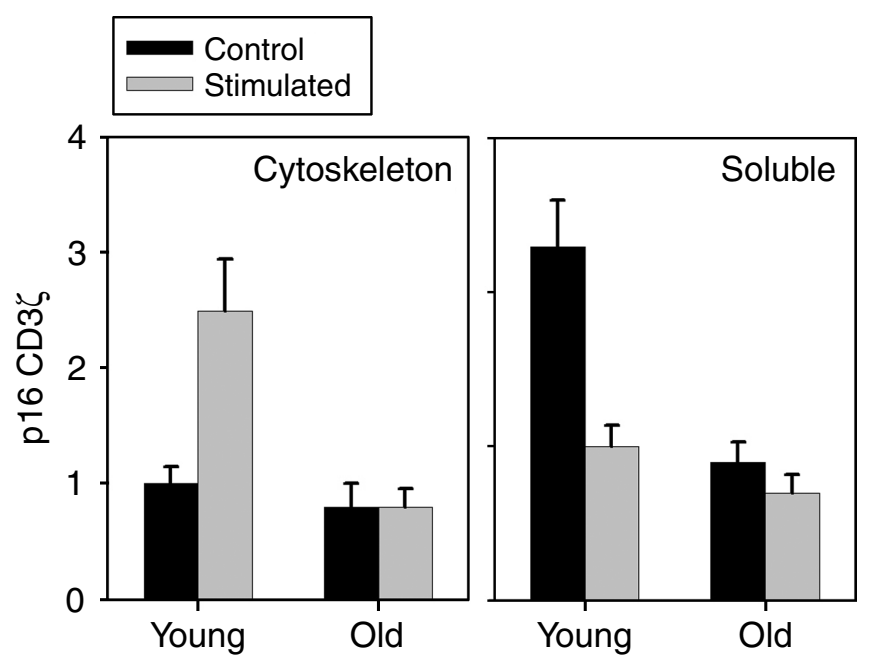

Fig. 6. Densitometric analysis of $\mathrm{CD} 3 \zeta$ levels, detected by immunoblotting, in cytoskeletal and detergent-soluble fractions of $\mathrm{CD}^{+}{ }^{+} \mathrm{T}$ cells either prior to or $5 \mathrm{~min}$ after stimulation with crosslinked antibodies to CD3, CD4, and CD28. Bars show mean and standard errors of the mean for five young and five old donors tested individually in two independent experiments; changes are significant $(\mathrm{P}<0.05)$ for young mice in both fractions but for old mice in neither fraction. Values are normalized so that 1 unit represents the level of the p21 form of $\mathrm{CD} 3 \zeta$ in the non-stimulated young $\mathrm{CD} 4{ }^{+} \mathrm{T}$-cell sample for each experiment. Data are from Garcia \& Miller (28). nological synapse organization and T-cell commitment to cytokine production and blast formation.

\section{Age-dependent changes in CD43 translocation and CD4 glycosylation patterns}

Activated $\mathrm{T}$ cells use cytoskeletal motors not only for assembly of elements of the immunological synapse but also for movement of surface molecules to the distal pole complex (DPC), i.e. to the area of the T-cell membrane opposite from the point of contact with the APC (29). CD43, a large, heavily glycosylated protein prominently represented at the T-cell surface, is among the molecules that are moved to the DPC after T-cell activation (30). The large size of the CD43 molecule, along with evidence that CD43 knockout mice are hyperresponsive in some tests of T-cell function (31, 32), has suggested that removal of CD43 from the area of immunological synapse formation might help to promote functional interaction between the T cell and APC.

To see if age-dependent changes in cytoskeletal function might hinder removal of CD43 from the zone of T cell/APC contact, we used confocal microscopy to count the number of $\mathrm{CD}^{+}{ }^{+} \mathrm{T}$ cells from young or old mice that were able to remove CD43 from the area of T-cell/APC contact using TCR-Tg T cells and peptide-bearing APCs. Fig. 7 shows an image of one such T-cell/APC conjugate in which CD43 (upper left panel) is excluded from the synapse area, identified (upper right panel) by staining for LAT. The proportion of $\mathrm{T}$ cells able to exclude CD43 from the synapse decreases dramatically with age (33) (Fig. 7), consistent with the idea that CD43 molecules might interfere with the T-cell/APC interactions in $\mathrm{T}$ cells from aged donors.

Analysis of CD43 glycosylation patterns (33) revealed another difference in CD43 properties between $\mathrm{T}$ cells from young and aged mice. Although immunoprecipitation data using an antibody specific for cytoplasmic determinants showed no difference in the total amount of CD43 in young and old $\mathrm{T}$ cells, flow cytometry showed an increase with age in expression of determinants detected by antibodies (S7 and 1 B 11) specific for the glycosylated forms of CD43. Although nearly all CD4 cells showed some expression of S7 and 1 B 11 determinants, the proportion of cells expressing higher levels of these two molecules increased substantially in the older mice. High-level expression was noted in both naïve and memory $\mathrm{T}$ cells (using CD44 levels as an index of memory cell differentiation), suggesting that the acquisition of the heavily glycosylated forms did not require a history of antigen exposure and clonal expansion to memory cells. Interestingly, 

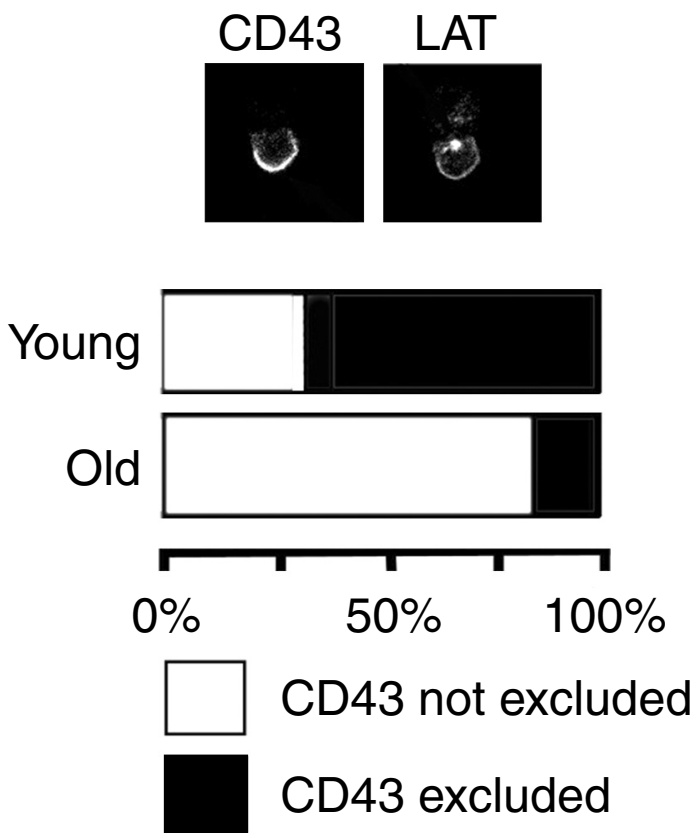

Fig. 7. Age-related decline in proportion of $\mathrm{CD}^{+}{ }^{+} \mathrm{T}$ cells that exclude CD43 from the immunological synapse. $\mathrm{CD}^{+}{ }^{+} \mathrm{T}$ cells from $\mathrm{T}$-cell receptor-transgenic mice were allowed to form complexes with APCs bearing agonist peptide (PCCF) and then were stained for both linker of activated $\mathrm{T}$ cell (LAT) and CD43. Top panel shows an example of a T-cell/antigen-presenting cell conjugate in which CD43 (left image) is excluded from the immunological synapse localized by LAT-fluorescent signal (right image). The bar graphic shows the proportion of $\mathrm{T}$ cells from young and old mice in which CD43 was excluded from the synapse (filled bar) or was not excluded (unfilled bar) (33).

$80 \%$ or more of the $\mathrm{CD}^{+}{ }^{+} \mathrm{T}$ cells with heavily glycosylated CD43 determinants were found to have high levels of surface P-glycoprotein, associated in previous studies with anergy among CD4M cells in both young and old mice (5-7). This finding suggested the hypothesis that the accumulation of heavily glycosylated forms of $\mathrm{CD} 43$ might contribute to the hyporesponsiveness of the P-glycoprotein-positive subsets of $\mathrm{CD}^{+}{ }^{+} \mathrm{T}$ cells, which become progressively more prominent in aging mice (3).

Enzymatic cleavage of surface glycoproteins restores synapse formation and improves activation of $\mathrm{T}$ cells from aged mice The increase with age in $\mathrm{CD} 43$ glycosylation together with the decline in the proportion of $\mathrm{T}$ cells that can exclude CD43 from the immunological synapse suggested a model in which glycosylated forms of CD43 contribute to the decline in activation of $\mathrm{T}$ cells from aged donors. As one test of this idea, we exposed $\mathrm{T}$ cells to an enzyme O-sialylglycoprotein endopeptidase (OSGE) that cleaves the protein backbone of molecules, such as CD43 that carry O-linked polysaccharide chains (33). The results (Fig. 8) showed that brief exposure to OSGE treatment restores the ability of $\mathrm{T}$ cells to form synapses when conjugated to peptide-bearing APCs. In cells not exposed to OSGE, positive responses were obtained from approximately $50 \%$ to $70 \%$ of the young $\mathrm{T}$ cells and $16 \%$ to $27 \%$ of the old T cells; OSGE had little or no effect on the proportion of positive $\mathrm{T}$ cells for young $\mathrm{CD} 4$ cells, but increased the proportion of responding old cells to a level nearly equivalent to that seen in untreated young cells. Similar results were seen when synapses were scored using antibodies to talin, F-actin, $\mathrm{CD} 3 \zeta$, or Grb2 (33). Treatment of the T cells with sialidase from Vibrio cholerae had no effect on synapse formation [not shown in Fig. 8, refer to Garcia \& Miller (33)], suggesting that the restoration of responsiveness was not due simply to removal of terminal sialic acid residues present on CD43 or other OSGE-sensitive T-cell surface glycoproteins. Furthermore, OSGE treatment of $\mathrm{CD}^{+}{ }^{+} \mathrm{T}$ cells from aged donors restored their ability to translocate talin to the APC contact zone in response to antagonist peptide (Fig. 8, right panel), showing that the enzyme treatment affects age-sensitive steps in T-cell/APC interaction that precede recognition of agonist peptide determinants by the TCR.

The effects of OSGE on aged $\mathrm{T}$ cells are not limited to synapse formation: OSGE-treated $\mathrm{CD} 4^{+} \mathrm{T}$ cells increase their ability to respond to anti-CD3 by activation of the CD25 and CD69 surface antigens (33), by increases in calcium signal generation, and by production of interleukin-2 (IL-2) and other cytokines (Berger et al., manuscript submitted). Fig. 9

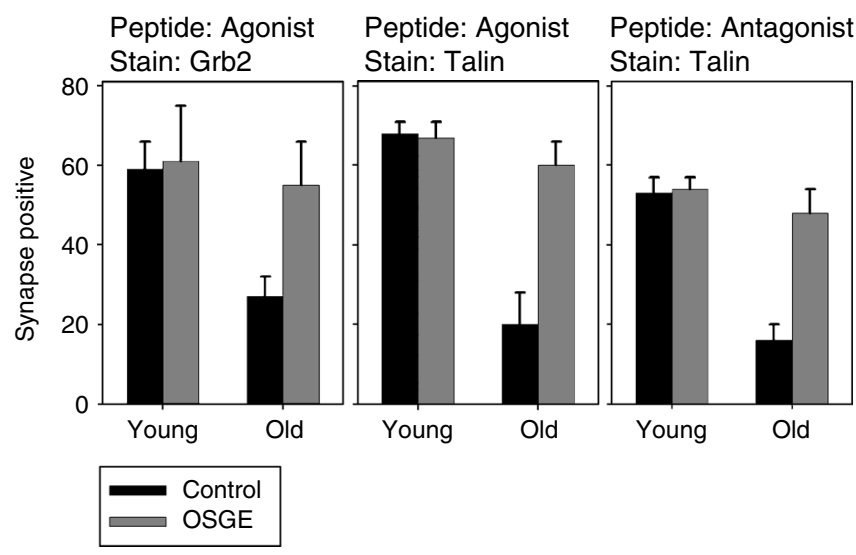

Fig. 8. O-sialylglycoprotein endopeptidase (OSGE) restores synapse formation in response to agonist or antagonist peptides in $\mathrm{CD}^{+} \mathrm{T}$ cells from old mice. Each bar shows mean and SD for experiments using six young and three aged donor mice, as the percentage of T-cell/ antigen-presenting cell (APC) conjugates that became synapse-positive using Grb2 (left panel) or talin (middle panel) as indicator for synapse formation in response to agonist peptide-loaded APCs, or which moved talin to the area of APC contact (right panel) when APCs were loaded with antagonist peptide fragments. Control samples (black bars) were processed in parallel omitting the treatment with OSGE. 
shows levels of CD69 expression in OSGE-treated and control CD 4 cells evaluated $6 \mathrm{~h}$ after activation by anti-CD3 antibody. $\mathrm{CD}^{+} \mathrm{T}$ cells from old donors (filled bars) are less responsive than young cells prior to treatment. Sialidase from Vibrio cholerae (VC) does not affect responsiveness of either young or old cells, but OSGE treatment increases the proportion of responding cells in both young and old mice. There is no age difference in levels of responsiveness after OSGE treatment, and both naïve and memory $\mathrm{T}$ cells (gated based on expression of CD44) show the effects of OSGE exposure (Fig.9, top and middle panels). The figure also depicts CD25 expression measured at $24 \mathrm{~h}$ after stimulation; in this case, naïve and memory cells could not be separately evaluated due to the stimulusinduced increase in CD44 levels. It is noteworthy that OSGE improves CD69 and CD25 activation of CD4 cells from young mice, even though it does not increase the proportion of young $\mathrm{CD}^{+} \mathrm{T}$ cells that can form immunological synapses in APC conjugates (Fig. 8); presumably this outcome is a consequence of OSGE-cleavage of surface molecules that would, if intact, interfere with stages in the activation process subsequent to or independent of synapse formation.

Fig. 9 also shows that a second sialidase, CP-sialidase, derived from Clostridia perfringens can mimic the effect of OSGE on CD69 and CD25 expression, and Sadighi Akha et al. (34) show that this reagent also increases calcium signal generation in $\mathrm{CD} 4^{+} \mathrm{T}$ cells of both young and old mice. This enzyme has similar but not identical substrate specificity with the sialidase from $V$. cholerae, shown to be ineffective both in the synapse assay (33) and in tests for T-cell activation antigens (Fig.9). Both sialidases cleave sugar/sugar bonds; in contrast, OSGE cleaves the peptide backbone of CD43 and other glycoproteins with Olinked glycan groups. More work will be needed to determine to what extent the ability of OSGE and C. perfringens sialidase to facilitate various stages in the T-cell activation cascade depends upon removal or modification of the same (or overlapping sets of) T-cell surface molecules.

\section{T-cell activation: current status and future goals}

The evidence reviewed above suggests a working hypothesis for future studies, in which activation defects of $\mathrm{T}$ cells from aged mice are attributed at least partly to hyperglycosylation of CD43 and other surface glycoproteins, coupled to or combined with alterations in the cytoskeletal machinery required to remove these bulky molecules from the area of interaction with the APC. The ability of OSGE treatment to restore calcium signals, synapse formation, and induction of surface antigens and cytokine production in $\mathrm{CD}^{+}{ }^{+} \mathrm{T}$ cells of aged mice suggests

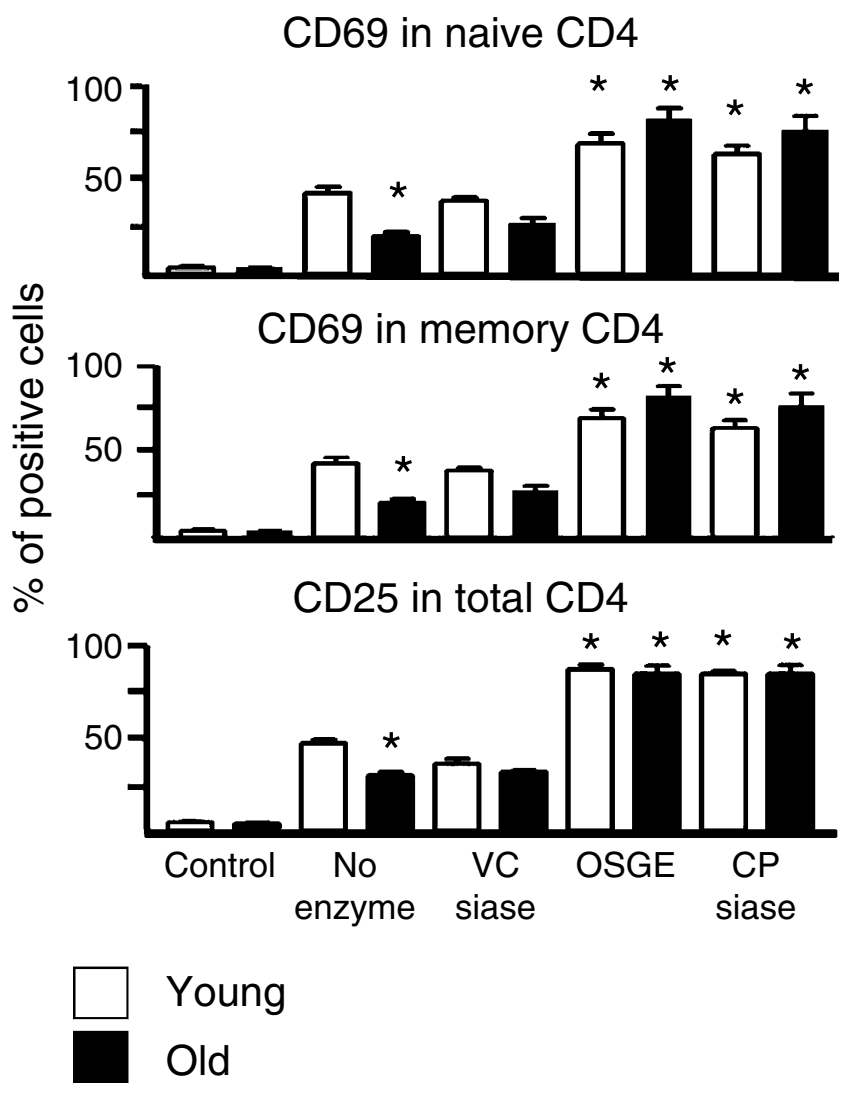

Fig. 9. Increased expression of CD69 and CD25 in $\mathrm{CD}^{+}{ }^{+} \mathrm{T}$ cells activated after treatment with OSGE or with sialidase (Siase) from Clostridia perfringens (CP). CD69 levels were evaluated $6 \mathrm{~h}$ after activation with anti-CD3 antibody and CD25 levels after $24 \mathrm{~h}$. Cells were gated for CD4 expression; for the CD69 study, they were also gated using antiCD44 for naïve (CD44 low) and memory (CD44 high) subsets. Each bar represents mean and standard errors of the mean for eight young (open bars) and four old mice. Asterisks indicate responses that are significantly different from the values for young $\mathrm{CD}^{+}{ }^{+} \mathrm{T}$ cells not exposed to OSGE or sialidase. 'Control' cultures, shown in the leftmost bars, represent cells in which isotype-matched antibody was substituted for the anti-CD3 antibody during the 6- or 24-h culture. Sialidase from Vibrio cholerae (VC siase) was used as an additional control and does not improve responsiveness (34).

that OSGE-sensitive molecules interfere with an early step or steps required for a wide range of downstream events in the activation cascade.

The mechanism by which OSGE improves function of T cells from aged mice is likely to be considerably more complex than those implied by this simplified model. We have preliminary data, for example, to show that OSGE can promote T-cell activation even when the $\mathrm{T}$ cells come from CD43 knockout mice (Berger, Garcia, Sadighi Akha et al., manuscript in preparation) suggesting strongly that OSGE can cleave other O-linked T-cell surface glycoproteins whose destruction facilitates T-cell activation. In addition, we have found that T cells from young and old mice differ substantially in patterns of 
glycosyltransferase levels and in patterns of binding of lectins specific for different polysaccharide bonds (34) as well as in relative levels of mRNA for specific glycosyltransferases (Berger et al., unpublished data). Thus, much more work will be needed to determine which T-cell surface molecules are differentially glycosylated in specific cell subsets in older mice and to learn which of these alterations leads to functional consequences.

\section{References}

1. Jackson AU, Fornes A, Galecki A, Miller RA, Burke DT. Multiple-trait quantitative trait loci analysis using a large mouse sibship. Genetics 1999; 151:785-795.

2. Miller RA, Jackson AU, Galecki AT, Burke DT. Genetic polymorphisms in mouse genes regulating age-sensitive and age-stable $\mathrm{T}$ cell subsets in mice. Genes Immun 2003;4: 30-39.

3. Witkowski JM, Miller RA. Increased function of P-glycoprotein in T lymphocytes of aging mice. J Immunol 1993;150:1296-1306.

4. Linton PJ, Haynes L, Klinman NR, Swain SL. Antigen-independent changes in naive CD4 T cells with aging. J Exp Med 1996;184: 1891-1900.

5. Bining N, Miller RA. Cytokine production by subsets of CD4 memory $\mathrm{T}$ cells differing in P-glycoprotein expression: effects of aging. J Gerontol A Biol Sci Med Sci 1997;52: B137-B145.

6. Witkowski JM, Miller RA. Calcium signal abnormalities in murine $\mathrm{T}$ lymphocytes that express the multidrug transporter P-glycoprotein. Mech Ageing Dev 1999;107:165-180.

7. Eisenbraun MD, Tamir A, Miller RA. Altered composition of the immunological synapse in an anergic, age-dependent memory $\mathrm{T}$ cell subset. J Immunol 2000;164:6105-6112.

8. Harper JM, Galecki AT, Burke DT, Pinkosky SL, Miller RA. Quantitative trait loci for insulin-like growth factor-I, leptin, thyroxine, and corticosterone in genetically heterogeneous mice. Physiol Genomics 2003; 15:44-51.

9. Wolf N, et al. Quantitative trait locus mapping for age-related cataract severity and synechia prevalence using four-way cross mice. Invest Ophthalmol Vis Sci 2004;45:1922-1929.

10. Volkman SK, Galecki AT, Burke DT, Miller RA, Goldstein SA. Quantitative trait loci that modulate femoral mechanical properties in a genetically heterogeneous mouse population. J Bone Miner Res 2004;19:1497-1505.

11. Jackson AU, Galecki AT, Chrisp C, Burke DT, Miller RA. Mouse loci associated with life span exhibit sex-specific and epistatic effects. J Gerontol A Biol Sci Med Sci 2002;57: B9-B15.
12. Miller RA, Chrisp C, Jackson AU, Galecki AT, Burke DT. Coordinated genetic control of neoplastic and nonneoplastic diseases in mice. J Gerontol A Biol Sci Med Sci 2002;57:B3-B8.

13. Miller RA, Chrisp C, Galecki A. CD4 memory T cell levels predict lifespan in genetically heterogeneous mice. FASEB J 1997;11:775-783.

14. Miller RA. Biomarkers of aging: prediction of longevity by using age-sensitive T-cell subset determinations in a middle-aged, genetically heterogeneous mouse population. J Gerontol 2001;56:B180-B186.

15. Miller RA, Chrisp C. T cell subset patterns that predict resistance to spontaneous lymphoma, mammary adenocarcinoma, and fibrosarcoma in mice. J Immunol 2002;169:1619-1625.

16. Miller RA, et al. Candidate biomarkers of aging: age-sensitive indices of immune and muscle function co-vary in genetically heterogeneous mice. J Gerontol A Biol Sci Med 1996;52:B39-B47.

17. Harper JM, Galecki AT, Burke DT, Miller RA. Body weight, hormones and T-cell subsets as predictors of lifespan in genetically heterogeneous mice. Mech Ageing Dev 2004;125:381-390.

18. Miller RA, Harper JM, Dysko RC, Durkee SJ, Austad SN. Longer life spans and delayed maturation in wild-derived mice. Exp Biol Med 2002;227:500-508.

19. Miller RA, Garcia GG, Kirk CJ, Witkowski JM. Early activation defects in $\mathrm{T}$ lymphocytes from old mice. Immunol Rev 1997;160: 79-90.

20. Kirk CJ, Miller RA. Analysis of Raf-1 activation in response to TCR activation and co-stimulation in murine T-lymphocytes: effect of age. Cell Immunol 1998;190:33-42.

21. Kirk CJ, Freilich AM, Miller RA. Age-related decline in activation of JNK by TCR and CD28-mediated signals in murine $T$ lymphocytes. Cell Immunol 1999;197: 75-82.

22. Kirk CJ, Miller RA. Age-sensitive and insensitive pathways leading to JNK activation in mouse CD4 T cells. Cell Immunol 1999;197:83-90.
23. Garcia GG, Miller RA. Increase in Zap-70 association with CD3 in CD4 $\mathrm{T}$ cells from old mice. Cell Immunol 1998;190:91-100

24. Tamir A, Eisenbraun MD, Garcia GG, Miller RA. Age-dependent alterations in the assembly of signal transduction complexes at the site of T cell/APC interaction. J Immunol 2000;165:1243-1251.

25. Yang D, Miller RA. Cluster formation by protein kinase $\mathrm{C}$ during murine $\mathrm{T}$ cell activation: effect of age. Cell Immunol 1999;195:28-36.

26. Garcia GG, Miller RA. Single-cell analyses reveal two defects in peptide-specific activation of naive $\mathrm{T}$ cells from aged mice. J Immunol 2001;166:3151-3157.

27. Monks CR, Kupfer H, Tamir I, Barlow A, Kupfer A. Selective modulation of protein kinase C-theta during T-cell activation. Nature 1997;385:83-86.

28. Garcia GG, Miller RA. Age-dependent defects in TCR-triggered cytoskeletal rearrangement in $\mathrm{CD}^{+}{ }^{+} \mathrm{T}$ cells. J Immunol 2002;169:50215027.

29. Cullinan P, Sperling AI, Burkhardt JK. The distal pole complex: a novel membrane domain distal to the immunological synapse. Immunol Rev 2002;189:111-122.

30. Allenspach EJ, et al. ERM-dependent movement of CD43 defines a novel protein complex distal to the immunological synapse. Immunity 2001;15:739-750.

31. Manjunath N, Correa M, Ardman M, Ardman B. Negative regulation of T-cell adhesion and activation by CD43. Nature 1995;377: 535-538.

32. Tong J, et al. CD43 regulation of $\mathrm{T}$ cell activation is not through steric inhibition of $\mathrm{T}$ cell-APC interactions but through an intracellular mechanism. J Exp Med 2004;199:1277-1283.

33. Garcia GG, Miller RA. Age-related defects in $\mathrm{CD} 4^{+} \mathrm{T}$ cell activation reversed by glycoprotein endopeptidase. Eur J Immunol 2003;33:3464-3472.

34. Garcia GG, Berger SB, Sadighi Akha AA, Miller RA. Age-associated changes in glycosylation of CD43 and CD45 on mouse CD4 T cells. Eur J Immunol 2005;35: 622-631. 